\title{
Route Optimization in Network Mobility
}

\section{Classification \& Comparison}

\author{
Md. Hasan Tareque \\ Department of Computer Science and Engineering \\ IBAIS University \\ Dhaka, Bangladesh
}

\author{
Ahmed Shoeb Al Hasan \\ Department of Computer Science and Engineering \\ Bangladesh University of Business \& Technology \\ Dhaka, Bangladesh
}

\begin{abstract}
NEtwork MObility (NEMO) controls mobility of a number of mobile nodes in a comprehensive way using one or more mobile routers. To choose a route optimization scheme, it is very important to have a quantitative comparison of the available route optimization schemes. The focus of this paper is to analyze the degree of Route Optimization (RO), deploy-ability and type of RO supported by each class in general. The comparison shows the differences among the schemes in terms of issues, such as additional header, signaling and memory requirement. We classify the schemes established on the basic method for route optimization, and equal the schemes based on protocol overhead, such as header overhead, amount of signaling, and memory requirements. Lastly the performance of the classes of different schemes has to be estimated under norms such as available bandwidth, topology of the mobile network and mobility type.
\end{abstract}

Keywords-Delegation; Hierarchical; Source Routing; BGPassisted; Network Mobility; Route Optimization

\section{INTRODUCTION}

The demand for wireless connectivity is rising now a days that it used both static and mobile IP-enabled devices. In the future, it may be common for several devices which are connected in a Local Area Network to move together.

Existing Internet is not designed to handle mobility due to IPs location-based addressing scheme where IP addresses are tied to geographical areas. A host moving between networks in different geographical areas needs to obtain a new IP address, and therefore, communication may become inefficient while maintaining reachability and session continuity. To overcome the inefficiency of current IP addressing, Internet Engineering Task Force (IETF) designed solutions such as Mobile IP (MIP) [1] and MIPv6 [2] to support mobility of a host. A summary of some of the host mobility protocols (including MIP and MIPv6) can be found in [3].

Handling mobility of a number of devices in a moving LAN or PAN using host mobility protocols for each device growths signalling overhead during handoff, power consumption and manageability. IETF developed NEtwork Mobility (NEMO) where one or more routers, called mobile routers, manage the mobility of all the hosts in a network. NEMO supports nested mobile network. IETF protracted MIPv6 to design NEMO Basic Support Protocol (NEMO BSP) [4] to grip network mobility, where hosts in a mobile network are reachable through a home agent.

\section{NEMO}

NEMO works by moving the mobility functionality from Mobile IP mobile nodes to a mobile router. The router is able to change its attachment point to the Internet in a manner that is transparent to attached nodes. Reduced Signalling, Increased manageability, Reduced power consumption, Conservation of bandwidth, Network Mobility \& Nested Mobile network are the main advantages of NEMO.

There are also some drawbacks in NEMO like inefficient routing which will increase end-to-end delay, bandwidth inefficiency and fragmentation. Due to encapsulation increase header overhead. Handoff latency may also rises due to NEMO.

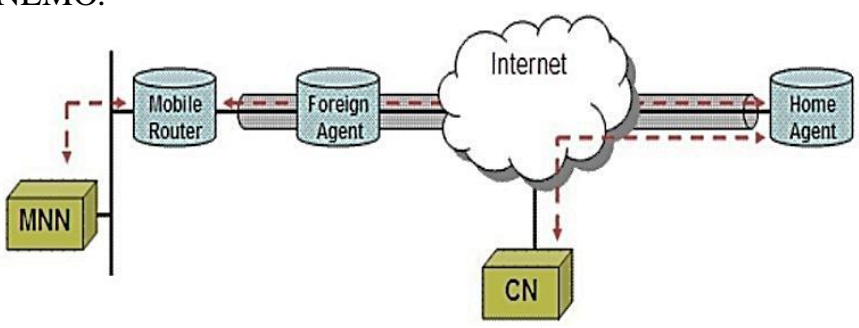

Fig. 1. Basic Idea of NEMO

In fig. 1, the simple idea of NEtwork MObility (NEMO) is been illustrated.

\section{A. Basic Design of NEMO}

For better understanding the basic NEMO structure there are some terminologies:

- Mobile Router $=$ MR .

- Mobile Network Node = MNN.

- $\quad$ Top Level Mobile Router = TLMR.

- Access Router = AR.

- $\quad$ Binding Update $=$ BU.

- Binding Acknowledgement = BA.

There are several types of MNNs:

- Local Fixed Node (LFN): Nodes which do not move with respect to the mobile network.

- Local Mobile Node (LMN): Nodes which usually reside in the mobile network but can move to other networks. 
- Visiting Mobile Node (VMN): Nodes which belong to another network but is currently attached to the mobile network.

- MR: An MNN can act as an MR to form a nested mobile network.

- Mobility Capable Nodes (MCNs): LMNs, VMNs and MRs implement mobility protocols; these are referred as MCNs.

- Home Network: The network to which a mobile network is usually connected.

- Home Agent (HA): An MR is registered with a router, called Home Agent, in its home network.

- Correspondent Node $(\mathrm{CN})$ : A node that communicates with MNNs.

- Home Address (HoA): Address through which TLMR is reachable in its home network. In fig. 2, the mobile network under MR1 is nested under TLMR's mobile network; MR1's mobile network thus has a nesting level of one.

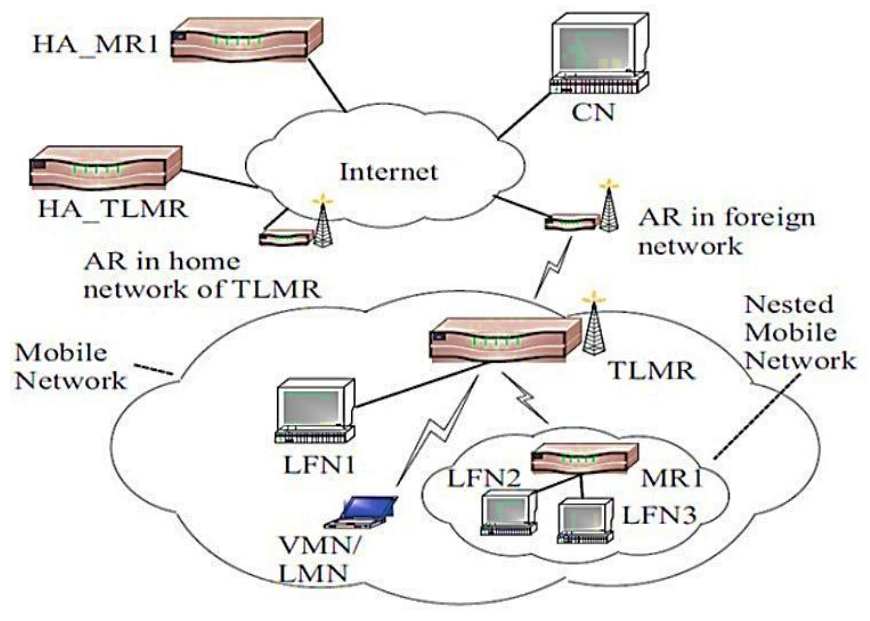

Fig. 2. Architecture of NEMO showing one level of nesting

- Care-of-Address (CoA): When the TLMR moves to a foreign network (any network other than home network), it obtains a new address from the foreign network.

An excellent style manual for science writers is [7].

\section{Routing Optimization (Ro)}

Route Optimization (RO) is solving the problem of inefficient route and header overhead. The basic principle of $\mathrm{RO}$ is to enable packets to directly reach the mobile network by avoiding multiple tunnels through home agents.

To trade off the gain of RO with the performance and applicability, several schemes have been proposed. In this section, we present the RO schemes and their challenges in and issues.

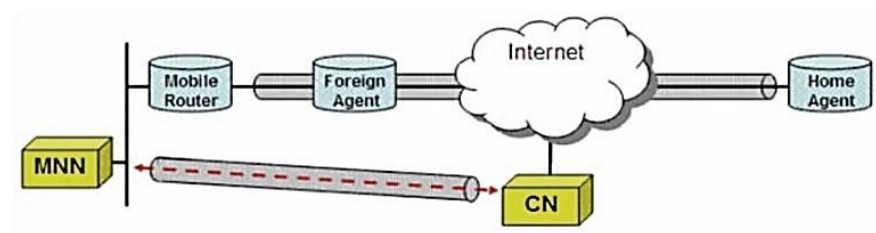

Fig. 3. Routing Optimization (RO)

In fig. 3, Shows how route optimization is been done in NEMO.

\section{A. Challenges in $R O$}

$\mathrm{RO}$ requires bypassing the HAs when packets are sent between $\mathrm{CN}$ and MNNs. Bypassing has given rise to the following two major challenges which have to be addressed by RO schemes:

- How can a packet destined to an MNN reach the TLMR attached to the foreign network to which the MNN is attached (directly or indirectly)?

- How is a packet routed inside the mobile network after reaching TLMR?

The challenge of RO in intra mobile network case (Intra $\mathrm{RO}$ ) is how to route packets between two MNNs without letting the packet outside the mobile network. Intra RO [5] is included because they also optimize route for communication between a $\mathrm{CN}$ and an MNN.

\section{B. Issues in $R O$}

There are several issues [6] that were raised in addition to header overhead and Intra RO those issues are given below.

\section{- Signaling}

When a mobile network moves, only the MR to which the movement is visible needs to perform signaling with its HA. Signaling packets competes with data packets for bandwidth not only inside the mobile network but also in the Internet.

\section{- Memory requirement}

The schemes have to maintain various state information, regarding the route and $\mathrm{CN}-\mathrm{MNN}$ pairs. Example: small sensors and PDAs.

- Degree of RO

In an effort to trade off issues, such as signaling, some schemes allow one or two levels of tunneling or some nonoptimality in the route between a $\mathrm{CN}$ and an MNN.

- Header overhead

Header overhead is the additional information that is put into the header for RO. Header overhead consumes bandwidth and increases chance of fragmentation.

- Intra RO

Route optimization between two MNNs within a mobile network is called Intra RO. With a focus on optimizing route between a $\mathrm{CN}$ and an $\mathrm{MNN}$, some of the schemes do not consider Intra RO. 
- Deploy-ability

Changes in mobility entities are tolerable because they are going to be introduced in the existing infrastructure if NEMO support is required. Changes in functionalities in hosts and routers in the existing infrastructure may not be easily applicable resulting in concern about deploy-ability issue.

- Location management

Location management is tracking the location of an MNN to ensure reachability and session continuity. In NEMO BSP and some RO schemes, location management is performed by HA.

- Location transparency

In NEMO BSP, MNNs except MRs and $\mathrm{CNs}$ are transparent.

\section{RO SCHEMES}

There are several RO schemes have been proposed to resolve the issues in RO. Based on approach used, the various RO schemes that have been proposed can be generally classified as:

- Delegation

- Hierarchical

- Source Routing

- Border Gateway Protocol (BGP)-assisted

The basic principle of each class, and a description and comparison of the schemes are discussed throughout the next section.

\section{A. Delegation Schemes}

In this class, prefix of the foreign network is delegated inside the mobile network. MCNs contain CoAs from the prefix and send BUs to respective HAs and CNs. So, any packet from $\mathrm{CN}$, addressed to $\mathrm{CoA}$, reached the foreign network without going through HAs.

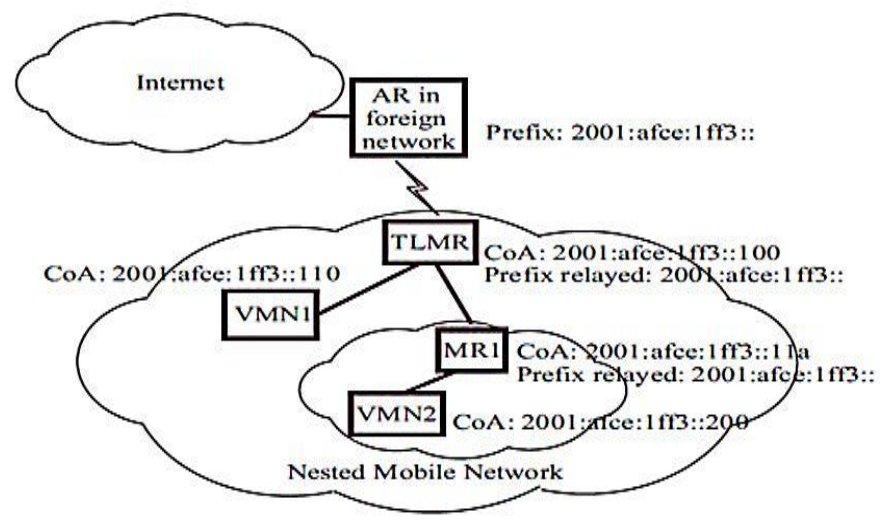

Fig. 4. Delegation approach for route optimization

In Fig. 4, prefix 2001:afce:1ff3:: is relayed by TLMR inside its mobile network. VMN1 and MR1 obtains CoA 2001:afce:1ff3:110 and 2001:afce:1ff3:11a, respectively; and MR1, in turn, relays the prefix inside its network.
The concept of prefix delegation is simple [7], and provides optimal route with low header overhead at the cost of sacrificing location transparency. $\mathrm{By}$, sending $\mathrm{BU}$ to $\mathrm{CN}$ requires additional signaling along with requirement of protocol sup- port from $\mathrm{CN}$, making the schemes difficult to execute. The schemes do not concentrate on Intra RO.

\section{B. Hierarchical Schemes}

In the hierarchical class, a packet, rather than traveling through all HAs, reaches the foreign network either from MNNs HA (first HA) or traveling only through HA of MNN and TLMR. Unlike delegation-based approach, an MR does not send its CoA to CNs. Rather; an MR sends TLMRs CoA or HoA to HA. CNs use MNNs HoA to send packets to an MNN. Packets, sent by $\mathrm{CN}$ to MNN, reach MNNs HA that tunnels the packets to TLMRs CoA or HoA. Packets, tunneled to CoA, directly reach the foreign network, whereas packets, tunneled to HoA, reach TLMRs HA that tunnels packets to TLMR. On reaching TLMR, packets are routed to MNN by MRs that maintains a routing table containing the mapping of MNNs prefix to next hop MR.

In Fig. 5, the abstract view of the hierarchical class is shown. TLMR CoA is passed to HA MR1 and HA VMN by MR1 and VMN, respectively. Also, MR1 and VMN send their CoAs to TLMR to enable forwarding inside the mobile network. Therefore, a packet sent to VMN will first reach HA VMN that tunnels the packet to the TLMR for forwarding towards the VMN. Thus, communication route is divided into two parts.

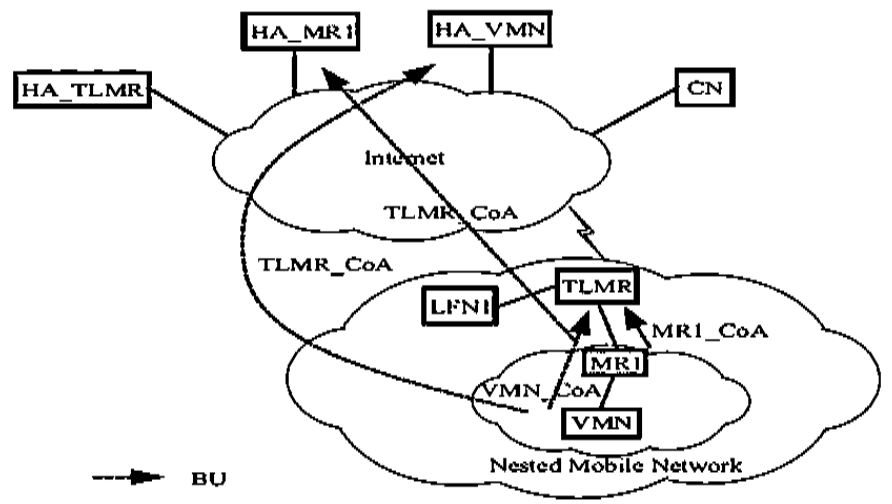

Fig. 5. Hierarchical approach for route optimization

- The route between TLMR and HA VMN.

- The route from the TLMR to VMN.

asAt least one tunnel always exists between the TLMR and HA VMN. The route between CN and MR1 is similar to that between $\mathrm{CN}$ and VMN.

The schemes in this class mainly differ in the use of TLMRs CoA or HoA for tunneling, techniques to convey TLMRs address to MRs, and routing of packets inside mobile network resulting in differences in signaling, memory requirement and degree of RO. Moreover, depending on the use of HoA or CoA of the TLMR, the number of tunnels used for communication differs among the schemes; number of 
tunnels affects degree of RO and header overhead. In addition, location management entities also vary among the schemes.

The schemes have the disadvantage of packets going through one or two tunnels, resulting in near optimal route and header overhead.

\section{Source Routing Schemes}

In this class, RO is achieved by sending the CoAs of MRs to the $\mathrm{CN}$ which, like source routing, inserts the CoAs in the packet header to reflect the nesting structure of the MRs. This however, results in increased header overhead. Packets from the $\mathrm{CN}$ reach TLMR in an optimal route (without going through HAs); routing within the mobile network is done using the CoAs in the packet header. Memory requirement for routing entries is low because each MR needs to keep track of only the attached MRs as next hop. Schemes in this class notify CN about the CoAs of MRs in various ways that will be detailed in the descriptions of the schemes. Notification of CoAs to CNs sacrifices location transparency and increases signaling. Methods of notifying the $\mathrm{CN}$ result in differences in signaling and overheads. Moreover, the schemes also have different memory requirement for routing packets inside the mobile network.

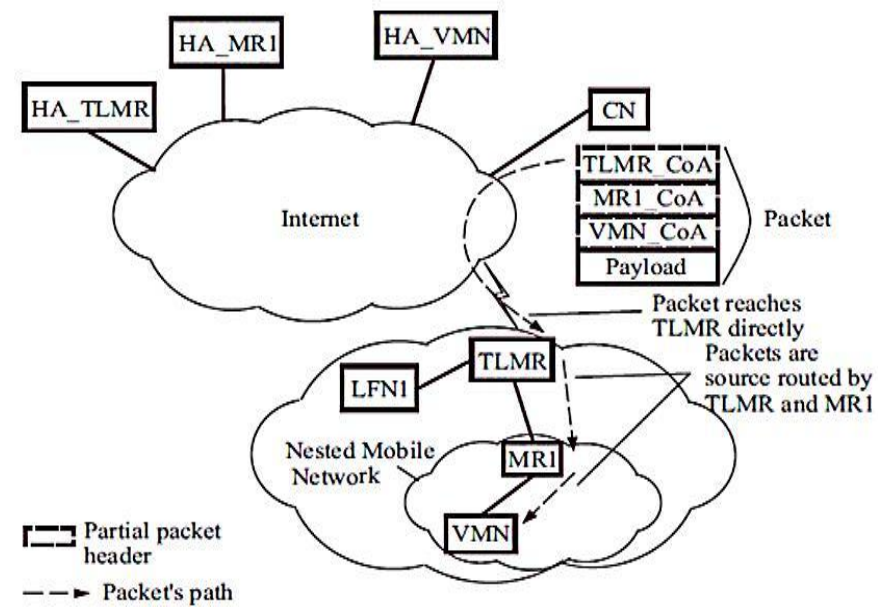

Fig. 6. Source routing approach

Fig 6. Shows the basic principle of the source routing approach where CoAs of TLMR, MR1 and VMN are inserted in packets. Packets, on reaching TLMR, are source routed (using the CoAs) inside the mobile network by TLMR and MR1.

\section{Border Gateway Protocol (BGP)-assisted Schemes}

The schemes in this class rely on BGP [8] for mobility management. When the mobile network moves, BGP routers are updated to make necessary changes in the routing tables by making forwarding entries for the prefix of the mobile network. Information regarding the change of route of the mobile network is signaled to few routers that exchange the information with peers using existing routing protocols in the Internet. Therefore, routers contain routing entries to route packets to the mobile network irrespective of its location, and are responsible for location management. Schemes in this class mainly differ in the number of external BGP updates generated, and incurring other overheads for managing Intra RO.

An abstract view of the approach used in this class has been shown in Fig. 7. When the TLMR joins the AR in the foreign network, AR injects a BGP update that maps TLMRs prefix $(1: 3: 1::)$ to ARs address $(1:: 2)$. BGP router3 in ARs network updates its peers (BGP router1 and BGP router2), accordingly. Therefore, packets sent by $\mathrm{CN}$ will reach a BGP router in its network, and will be forwarded to the appropriate BGP routers network where the mobile network resides.

The major advantage of the schemes in this class is the use of no new entity for mobility management. Moreover, CNs are transparent to the change location (managed by BGP routers) of the MNNs. On the other hand, these schemes will produce a storm of updates.

This trade of also requires packets always traveling through one or more of some designated routers resulting in near optimal route.

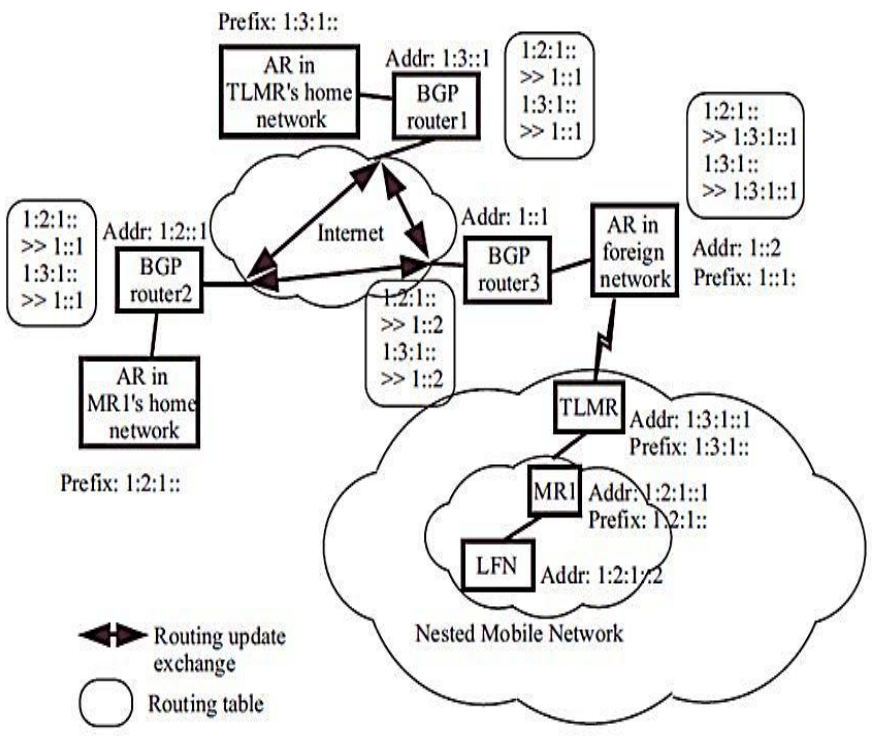

Fig. 7. BGP-assisted approach.

\section{E. Miscellaneous Schemes}

This section includes RO schemes that do not fall into any of the previous classes described. The techniques, used for RO in the schemes presented in this section, are different than the basic techniques used for RO.

a) Optimized Route Cache (ORC) - based: An approach [9] where the MR sends BU to a router in the CNs network, and to the MR attached above (parent MR). A major disadvantage of ORC is that it optimizes route for only one level of nesting. Although route from $\mathrm{CNs}$ to $\mathrm{MNNs}$ is similar to that in hierarchical class for one level of nesting, it is different when the nesting level increases. In hierarchical class, TLMRs HoA or CoA is not conveyed to the nested MRs. Therefore, we have placed this scheme separately in this section

b) Recursive $B U(R B U)$ - based: A RO scheme [10] where BUs, sent by MRs to $\mathrm{CN}$, are used to recursively process the 
binding table at $\mathrm{CN}$ to maintain a route to TLMR. Memory requirement for routing will be low when routes are discovered dynamically. Signaling in this scheme is high.

c) $A O D V$ - based: The route between a HA and an MR is established using AODV protocol [11]. The scheme appears to be very simple; yet, it requires all routers in the Internet, and HA to support AODV resulting the scheme difficult to deploy. Moreover, the scheme involves one tunnel for communication along with overhead of burst of messages in the Internet during handoff due to broadcast of AODV messages. Although AODV is a protocol for Ad hoc networks, we do not include AODV-based scheme in delegation class under Ad hoc-based scheme due to the following reason: The basic principle used in Ad hoc- based scheme is to obtain a CoA from the foreign network prefix contrasting the obtaining of CoA from MRs prefix in the AODV-based scheme.

TABLE I.

A COMPARISON AMONG DIFFERENT CLASSES

\begin{tabular}{ccccccc}
\hline Class & Degree of RO & Intra RO & Signalling & Header Overhead & Deployability & Location Transparency \\
\hline & & & & & & \\
Delegation & Optimal & No & High & Low & Difficult & No \\
Hierarchical & Near optimal & Yes & Low & Medium & Easy & Yes \\
Source routing & Optimal & No & High & High & Difficult & No \\
BGP-assisted & Near optimal & Yes & Low & Low & Difficult & Yes \\
& & & & & & \\
\hline
\end{tabular}

\section{ANALYSIS}

In this section, we perform comparison of different schemes which was discussed above.

- The comparisons show that hierarchical scheme are easier to deploy, and also supports efficient intra mobile network communication in the wired network.

- Delegation-based and BGP-assisted schemes suites the client-server type communication that prevails in the existing Internet.

- Delegation approach is simple, do not introduce any additional overhead on Internet routing, and optimize route completely.

- BGP-assisted approach supports Intra RO, and requires fewer supports from infrastructure.

- Source routing approach is not suitable for mobile networks having higher nesting levels due to higher header overhead that consumes bandwidth which in wireless environment.

- There is a comparison shown in Table I among different schemes [Delegation, Hierarchical, Source Routing, BGP- assisted]

\section{FUTURE RESEARCH}

Although a considerable amount of research has been carried out in NEMO, there are still lots of issues where future research can be possible.

- Further Research is required to determine the performance of the RO schemes with change in network topology.

- Most of the RO schemes incur additional signaling over bandwidth limited wireless channels. This contradicts one of the initial objectives of NEMO as a scheme to reduce signaling over wireless channels by letting the mobile router carry out the signaling on behalf of all the nodes. To reduce the signaling, a constant level (one or two) of tunneling can be allowed.

Again update for all the CNs and HAs can possible with a single BU. This is done by letting the CNs and HAs join a multicast group when they join the mobile network. RO schemes can be analyzed to find suitable schemes based on the architecture of the mobile network, availability of bandwidth, and mobility pattern. To reduce the signaling, a constant level of tunneling can be allowed. This has been done in some of the schemes in hierarchical class, and can be adopted in other schemes dynamically on ad hoc basis.

- Considering handoff performance [12] [13] along with any RO scheme is important.

- Security threats [14] also need to be considered in conjunction with RO.

\section{CONCLUSION}

The mobile network that supports NEMO is foreseeable in mobile platforms such as car, bus, train, air-plane, etc. The use of NEMO BSP [4] gives more assistances than MIPv6 [2] in mobile platforms. The restrictions of NEMO can be addressed by RO schemes. Although RO schemes address the problems of NEMO, ensuring QoS is great challenge in various internet applications.

In this paper, we present classification and comparison among the RO schemes for NEMO. The number of RO schemes reported in this article indicates the exhausting and diverse efforts for $\mathrm{RO}$, and therefore, requires a quantitative evaluation of the RO schemes to determine their suitability and adaptability to the existing Internet infrastructure.

The comparison among the schemes within each class reveals the differences among the schemes in more depth. Moreover, signaling and memory requirement depend on the number and types of MNNs in the mobile network, and 
therefore, might guide the selection of the schemes. Hence, the evaluation under various parameters is required to determine the suitability of the schemes. To apply the RO schemes to real-world applications and enable their wide deployment, protocol overheads, such as header overhead and signaling need to be reduced.

The internet applications can be classified into real-time and non-real-time applications. The real-time applications are real-time interactive audio and video applications; one-to-many streaming of real-time audio and video applications; streaming of stored audio and video applications. The non-real time applications are file transfer, web access, e-mail, etc. As the QoS (Quality of Service) requirements for each application can vary from each other, suitable selection of existing RO schemes was done. It is obvious that most of the RO schemes are not considered all QoS parameters such as delay, jitter, and bandwidth and packet loss. In future, it is necessary to focus on these QoS parameters. The limitations of each and every scheme can be further studied with respect to QoS requirements.

\section{ACKNOWLEDGMENT}

A special thanks to Dr. Md. Shohrab Hossain for giving us a chance to work with this topic. Also a superior thanks goes to Abu Zafar M. Shahriar, Mohammed Atiquzzaman, there work was really helpful.

\section{REFERENCES}

[1] C. Perkins, "IP mobility support for IPv4," RFC 3220, Jan 2002.

[2] D. B. Johnson, C. E. Parkins, and J. Arkko, "Mobility support in IPv6," RFC 3775, Jun 2004.
[3] A. Conta and S. Deering, "Generic packet tunneling in IPv6 specifications," RFC 2473, Dec 1998.

[4] V. Devarapalli, R. Wakikawa, A. Petrescu, and P. Thubert, "NEtwork MObility (NEMO) basic support protocol," RFC 3963, Jan 2005.

[5] C. Ng, P. Thubert, M. Watari, and F. Zhao, "Network mobility route optimization problem statement," RFC 4888, Jul 2007.

[6] C. Ng, F. Zhao, M. Watari, and P. Thubert, "Network mobility route optimization problem statement," RFC 4889, Jul 2007.

[7] K. Lee, J. Park, and H. Kim, "Route optimization for mobile nodes in mobile network based on prefix delegation," IEEE 58th Vehicular Technology Conference, pp. 2035-2038, Oct 6-9 2003.

[8] Y. Rekhter, T. Li, and S. Hares, "A border gateway protocol 4 (bgp-4)," RFC 4271, Jan 2006.

[9] R. Wakikawa, S. Koshiba, K. Uehara, and J. Murai, "Orc: Optimize route cache management protocol for network mobility," 10th International Conference on Telecommunications, pp. 1194-1200, Feb 23 - Mar 12003.

[10] H. Cho, E. K. Paik, and Y. Choi, "Rbu+: Recursive binding update for end-to-end route optimization in nested mobile networks," 7th IEEE International Conference on High Speed Networks and Multimedia Communications, pp. 468-478, Jun 30 - Jul 22004.

[11] R. Cuevas, A. Cabellos-Aparicio, A. Cuevas, J. Domingo-Pascual, and A. Azcorra, "fp2p-hn: A p2p-based route optimization architecture for mobile ip-based community networks," Computer Networks, pp. 528540, Mar 2009.

[12] H. Petander, E. Perera, K. Lan, and A. Seneviratne, "Measuring and improving the performance of network mobility management in IPv6 networks," IEEE J. Sel. Areas Commiun, pp. 1671-1681, Sep 2006.

[13] H. K. Ryu, D. H. Kim, Y. Z. Cho, K. W. Lee, and H. D. Park, "Improved handoff scheme for supporting network mobility in nested mobile networks," International Conference on Computational Science and Its Applications, pp. 378-387, May 9-12 2005.

[14] S. Jung, F. Zhao, and H. Kim, "Threat analysis on network mobility (NEMO)." Sixth International Conference on Information and Communications Security, Oct 27-29 2004. 